\title{
STIM2 regulates activity-dependent AMPA receptor trafficking and plasticity at hippocampal synapses
}

Authors: Kenrick An Fu Yap ${ }^{1,+}$, Mahesh Shivarama Shetty ${ }^{2,+}$, Gisela Garcia-Alvarez ${ }^{1,+}$, Bo Lu ${ }^{1}$ Masatsugu Oh-Hora ${ }^{3}$, Sreedharan Sajikumar ${ }^{2, *}$ and Marc Fivaz ${ }^{1,2 *}$

${ }^{1}$ DUKE-NUS Medical School. Program in Neuroscience and Behavioral Disorders, Singapore.

${ }^{2}$ Department of Physiology, Yong Loo Lin School of Medicine, National University of Singapore. ${ }^{3}$ Division of Molecular Immunology, Medical Institute of Bioregulation, Kyushu University, Japan.

${ }^{+}$equal contribution

${ }^{*}$ Correspondence:

Marc Fivaz (marc.fivaz@duke-nus.edu.sg) and Sreedharan Sajikumar (phssks@nus.edu.sg) 


\begin{abstract}
STIM2 is an integral membrane protein of the endoplasmic reticulum (ER) that regulates the activity of plasma membrane (PM) channels at ER-PM contact sites. Recent studies show that STIM2 promotes spine maturation and surface expression of the AMPA receptor (AMPAR) subunit GluA1, hinting to a probable role in synaptic plasticity. Here, we used a Stim2 cKO mouse line to explore the function of STIM2 in early forms of Long-Term Potentiation (E-LTP) and Depression (E-LTD), two widely-studied models of synaptic plasticity implicated in information storage. We found that STIM2 is required for the stable expression of both E-LTP and E- LTD at CA3-CA1 hippocampal synapses. Altered plasticity in Stim2 cKO mice is associated with subtle alterations in the shape and density of dendritic spines in CA1 neurons. Further, surface delivery of GluA1 in response to LTP-inducing chemical manipulations was markedly reduced in excitatory neurons derived from Stim2 cKO mice. In addition, NMDAinduced GluA1 endocytosis, which underlies LTD, was impaired in Stim2 cKO neurons. We conclude that STIM2 facilitates synaptic delivery and removal of AMPARs and regulates activity-dependent changes in synaptic strength through a unique mode of communication between the ER and the synapse.
\end{abstract}

\title{
Keywords
}

Endoplasmic reticulum, excitatory synapse, plasticity, memory, long-term potentiation, longterm depression, AMPA receptors. 


\section{Introduction}

The endoplasmic reticulum (ER) influences synaptic transmission, plasticity and pathophysiology, through its ability to shape synaptic $\mathrm{Ca}^{2+}$ signals, regulate the assembly and trafficking of synaptic receptors and control cellular responses to stress ${ }^{1}$. Mobilization of $\mathrm{Ca}^{2+}$ from the ER, in response to chemical or electrical inputs, is one central mechanism by which this organelle regulates synaptic transmission and plasticity ${ }^{2}$. In most cell types, release of $\mathrm{ER} \mathrm{Ca}^{2+}$ triggers Store-Operated $\mathrm{Ca}^{2+}$ entry (SOCE), a form of $\mathrm{Ca}^{2+}$ influx which is regulated by the ERresident STIM proteins ${ }^{3}$. While SOCE is the predominant $\mathrm{Ca}^{2+}$ influx pathway in non-excitable cells ${ }^{4}$, the magnitude and relevance of SOCE in neurons remains a topic of debate ${ }^{5-7}$. Recent studies, however, reported SOCE responses in dendritic spines ${ }^{8,9}$, small dendritic protrusions at the tip of which terminate most excitatory inputs. Synaptic SOCE is STIM2-dependent, has been linked to spine maturation and is dysregulated in mouse models of Alzheimer's and Huntington's disease ${ }^{8-11}$.

On the other hand, STIM2 has also been proposed to function in a SOCE-independent manner by regulating phosphorylation and surface expression of the AMPAR ${ }^{7}$, the main glutamate receptor mediating fast excitatory neurotransmission in the brain. In particular, STIM2 promotes phosphorylation of Ser845 in the c-terminal tail of the AMPAR subunit GluA1 ${ }^{7,12}$. Phosphorylation of GluA1 on Ser845 regulates activity-dependent trafficking and synaptic insertion of the AMPAR ${ }^{13,14}$, and influences its channel properties ${ }^{15}$. Consistent with a function of STIM2 at the synapse, STIM2 is found in approximately half of hippocampal spines ${ }^{7,8}$ and presumably operates at contact sites between the ER and the dendritic PM to regulate the neuronal SOCE channel (yet to be identified) ${ }^{8}$ the AMPAR ${ }^{7}$, the postsynaptic voltage-gated $\mathrm{Ca}^{2+}$ channel Cav1.2 ${ }^{5,16}$ and possibly other effectors.

Activity-dependent remodeling of dendritic spines and trafficking of the AMPAR to and away from the synapse are two hallmarks of synaptic plasticity in the hippocampus, neocortex and other brain regions. LTP at many different central synapses is mediated by recruitment of GluA1-containing AMPARs to the post-synaptic membrane ${ }^{17-19}$ and is associated with rapid increase in dendritic spine size ${ }^{20}$. Conversely, LTD involves endocytic removal of the AMPAR from the synapse and spine shrinkage ${ }^{21}$. Thus, synaptic efficacy at glutamatergic synapses is tightly correlated to spine size and AMPAR content. The impact of STIM2 on spine morphogenesis and its role in AMPAR trafficking makes it an obvious candidate for plasticity, 
particularly in the hippocampus, where STIM2 is most abundant ${ }^{22}$. We thus examined the effect of STIM2 on these forms of synaptic plasticity at CA3-CA1 synapses in a Stim2 cKO mouse. Our results show that STIM2 is required for the stable expression of both E-LTP and E-LTD and suggest that abnormal trafficking of the AMPAR is responsible for impaired plasticity in these mice. We propose that STIM2-dependent ER-to-synapse signaling is a novel post-synaptic mechanism underlying activity-dependent changes of synaptic strength occurring during the initial phase of plasticity.

\section{Material and methods}

\section{DNA constructs, lentiviruses and Antibodies.}

The lentiviral vectors pFUGW (GFP, \#14883) and pLM-CMV-R-Cre (mCherry-P2A-Cre, \#14883) were from addgene. pFUmChW (mCherry) was made by replacing GFP by mCherry in the pFUGW vector and has been described before ${ }^{7}$. Lentiviral particles were produced according to a protocol adapted from ${ }^{23,24}$. Briefly, the lenviral construct of interest was co-transfected in HEK293T cells together with the envelope (VSV-G) and packaging $(\Delta 8.9)$ vectors. After 48 hrs, the supernatant was cleared by centrifugation at $1000 \mathrm{~g}$ for $3 \mathrm{~min}$ and filtered through a $0.22 \mu \mathrm{m}$ filter top. For in vivo injections, viruses were concentrated by ultra-centrifugation at $65,000 \mathrm{~g}$ and $4{ }^{\circ} \mathrm{C}$ for $2 \mathrm{hrs}$, followed by re-suspension in PBS, layering on top of a $0.5 \mathrm{ml} 20 \%$ sucrose cushion and re-ultra-centrifugation at $65,000 \mathrm{~g}$ and $4{ }^{\circ} \mathrm{C}$ for $2 \mathrm{hrs}$. The resulting pellet was resuspended in $100 \mu \mathrm{PBS}$, aliquoted, and stored at $-80^{\circ} \mathrm{C}$. The concentrated pFUGW virus used in this study had a titre of $2 \times 10^{13} \mathrm{TU} / \mathrm{ml}$. For transduction of neuron cultures, we used a multiplicity of infection (MOI) typically between 2 and 3. The $\alpha$ GluA1 Ab (\#182 011) was from Synaptic Systems. The STIM2 and STIM1 Abs were from Cell Signaling (\#4917S) and ProSci (\#4119) respectively. The p-Ser845 (\#04-1073) and p-Ser831 (04-823) GluA1 Abs were from Millipore.

\section{Mouse lines}

Stim2 ${ }^{f l / f l}$ mice ${ }^{25}$ were crossed to B6.Cg-Tg(CaMKII $\alpha$-Cre $)$ T29-1Stl/J mice ${ }^{26}$. Floxed Stim2 males heterozygous for Cre (Stim2 $2^{\text {fl/fl. }}$ CaMKII $\left.\alpha-C r e^{+/}\right)$were then mated with Stim2 ${ }^{\mathrm{fl} / / \mathrm{fl}}$;CaMKII $\alpha$-Cre $\mathrm{Ca}^{-/-}$females to produce litters consisting of Stim2 $2^{\mathrm{fl} / \mathrm{fl}} ; \mathrm{Cre}^{+/-}($Stim2 $\mathrm{cKO})$ and Stim2 ${ }^{\mathrm{fl} / \mathrm{fl}} ; \mathrm{Cre}^{-/-}$(control littermates). Crossing CaMKIIa-Cre mice to a conditional tdTomato Cre 
reporter line ${ }^{27}$ showed that Cre recombinase is expressed in virtually all CA1 hippocampal neurons 90 days after birth (not shown, but see ${ }^{12}$ ). All animal procedures were approved by the Singhealth Institutional Animal Care and Use Committee (IACUC) of Singapore (protocol \# 2015/SHS/1096).

\section{Electrophysiology}

All animal procedures were carried out in accordance with protocol 072/12 approved by the IACUC of the National University of Singapore. A total of $15\left(\right.$ Stim2 $^{\mathrm{fl} / \mathrm{fl}} ; \mathrm{CaMKII} \alpha-\mathrm{Cre}^{+/-}=8$ and $\mathrm{Stim}_{2}{ }^{\mathrm{fl} / \mathrm{fl}} ; \mathrm{CaMKII} \alpha-\mathrm{Cr}^{-/-}=7$ ) animals were used in the study. After $\mathrm{CO}_{2}$ anaesthetization, mice were decapitated and the brains were quickly placed in $4^{\circ} \mathrm{C}$ artificial cerebrospinal fluid (ACSF) containing (in mM): $124 \mathrm{NaCl}, 4.9 \mathrm{KCl}, 1.2 \mathrm{KH}_{2} \mathrm{PO}_{4}, 2 \mathrm{MgSO}_{4}, 2 \mathrm{CaCl}_{2}, 24.6 \mathrm{NaHCO}_{3}$, and 10 D-glucose. The $\mathrm{pH}$ of ACSF was between 7.3-7.4 when bubbled with 95\% oxygen and 5\% carbon dioxide (carbogen). Transverse hippocampal slices ( $400 \mu \mathrm{m}$-thick) were prepared from the right hippocampus using a manual tissue chopper (Stoelting), transferred onto nylon net in an interface chamber (Scientific Systems Design, Canada) and incubated at $32^{\circ} \mathrm{C}$ at an ACSF flow rate of $1 \mathrm{~mL} / \mathrm{min}$ and carbogen consumption of $16 \mathrm{l} / \mathrm{h}$. The whole process, from anaesthetization to the transfer of slices to the chamber, was carried within 5 minutes. Slices were incubated for 2 hours in the chamber before proceeding with electrode location. In all experiments, 2 monopolar lacquer-coated, stainless-steel electrodes (5 M 2 ; AM Systems, Carlsborg) were positioned at an adequate distance within the stratum radiatum of the CA1 region, one to stimulate the Schaffer collaterals and another to record the fEPSP responses from the apical dendrites. The signals were amplified by a differential amplifier (Model 1700, AM Systems) and digitized using a CED 1401 analog-to-digital converter (Cambridge Electronic Design, UK) and monitored online with custom-made software. After incubation period, an input-output curve (afferent stimulation vs. fEPSP slope) was plotted and the test stimulus intensity for each slice was set to obtain fEPSP slope $40-50 \%$ of its maximal response. Four $0.2-\mathrm{Hz}$ biphasic constant-current pulses $(0.1$ $\mathrm{ms} /$ polarity) were used for baseline recording and testing at each time point. In all experiments, a stable baseline was recorded for at least 30 minutes before LTP/LTD induction. For LTP induction, a weak tetanization protocol (WTET) consisting of a single high frequency stimulation ( $100 \mathrm{~Hz}, 21$ biphasic constant current pulses, single burst, $0.2 \mathrm{~ms}$ pulse duration) was used. For LTD induction, a weak low-frequency protocol (WLFS) consisting of 900 pulses at a 
frequency of $1 \mathrm{~Hz}$, impulse duration of $0.2 \mathrm{~ms}$ per half-wave, with a total number of stimuli of 900 was used ${ }^{12}$. The initial slopes of fEPSPs were expressed as percentages of baseline averages. The time-matched, normalized data were averaged across replicate experiments and expressed as mean \pm SEM. Statistical analysis was performed with GraphPad Prism software. Wilcoxon signed rank test was used for within group comparisons and Mann-Whitney-U-test, when compared between groups. Statistical significance was assumed at $p<0.05$.

\section{Stereotaxic Injection}

Male mice aged 3 to 3.5 months were used. Prior to the surgery, the mouse was anaesthetized with a Ketamine $(50 \mathrm{mg} / \mathrm{kg})$ and Xylazine $(5 \mathrm{mg} / \mathrm{kg})$ cocktail before being mounted on a surgical platform equipped with head clamps. The skull was then exposed to visualize the bregma and lambda, and hydrogen peroxide $\left(\mathrm{H}_{2} \mathrm{O}_{2}\right)$ was applied to sterilize the surgical site. Small holes into the skull were created using an electric drill, targeting the dentate gyrus and CA1 hippocampal regions located in reference to the bregma $(-2.0 \mathrm{~mm}$ anterior/posterior, $\pm 1.8 \mathrm{~mm}$ lateral/medial, and -2.0 ventral/dorsal). $1.5 \mu$ of virus was injected into the hippocampi using a Hamilton syringe and needle at a rate of $0.25 \mu \mathrm{l} / \mathrm{min}$, which was controlled automatically by the stereotaxic apparatus. Following injections, the incision site was sutured using surgical thread to close the wound and the mouse was returned to a pre-warmed cage. The mouse will be kept for at least 2 weeks to recover.

\section{Brain Sectioning and Image Acquisition}

Two weeks after lentiviral injections, the mice were anaesthetized with pentobarbital and perfused with PBS, and subsequently 4\% paraformaldehyde (PFA) in PBS (pH 7.4). After dissecting out the brains, they were post-fixed in $4 \%$ PFA at $4^{\circ} \mathrm{C}$ overnight and cryoprotected in $15 \%$ and then $30 \%$ sucrose at $4{ }^{\circ} \mathrm{C}$ over two days. Coronal sections of $50 \mu \mathrm{m}$ thick were cut on a freezing microtome and stored in PBS. Free floating sections were then permeabilized with $0.25 \%$ Triton X-100 in PBS for 30 min and blocked with 2\% BSA, 10\% horse serum and $0.25 \%$ Triton X-100 in PBS for 30 min, followed by incubation in chicken anti-GFP antibody (1:1000, AbCam ab13970) at $4{ }^{\circ} \mathrm{C}$ overnight. The sections were next incubated with goat antichicken 488 secondary antibody (Alexa Fluor, Life Technologies) for $1 \mathrm{hr}$ at room temperature, before they were mounted on glass slides with $0.2 \%$ gelatine in $50 \mathrm{mM}$ Tris- $\mathrm{HCl}(\mathrm{pH} 7.5)$, and 
coverslips using FluorSave (CalBiochem). Slides were imaged with an inverted Zeiss LSM 710 laser scanning confocal microscope driven by Zen 2010B software. A 10X air objective was used to take tiled images of the coronal section and a $63 \mathrm{X}$ oil objective was used to take Z-stacks of dendritic segments. The sample was excited with $488 \mathrm{~nm}$ solid-state lasers.

\section{Analysis of dendritic spines}

Spines were detected, scored and classified into mushroom, stubby, or thin using the default algorithm from NeuronStudio software ${ }^{28}$. Only secondary and tertiary apical dendrites were included in the analysis.

\section{Chemical LTP, LTD and surface biotinylation}

Stim $2^{f / f l}$ cortical neurons were prepared as previously described ${ }^{7}$ and transduced with mCherryP2A-Cre or mCherry. Neuron cultures (DIV 21) were transferred to $\mathrm{Mg}^{2+}$-free ACSF and exposed to the following treatments. Forsk-LTP: forsk/rolipr $(50 / 0.1 \mu \mathrm{M})$ for $30 \mathrm{~min}$ at $37^{\circ} \mathrm{C}$. Glyc-LTP: glycine $(200 \mu \mathrm{M})$, bicuculline $(20 \mu \mathrm{M})$ and strychnine $(3 \mu \mathrm{M})$ for $15 \mathrm{~min}$ at $37^{\circ} \mathrm{C}$. NMDA-LTD: NMDA $(50 \mu \mathrm{M}) 3 \mathrm{~min}$ at $37^{\circ} \mathrm{C}$. As control, cells were treated with DMSO (vehicle) alone. Neuron cultures were then rinsed twice with ice-cold PBS and incubated with sulfo-NHS-SS-biotin (Pierce) for $30 \mathrm{~min} 4^{\circ} \mathrm{C}$ on a shaker. Cells were then quenched in PBScontaining $25 \mathrm{mM}$ Tris- $\mathrm{HCl}(\mathrm{pH} 7.2)$ and lysed in ice-cold RIPA buffer. For avidin pulldowns, Neutravidin beads (Pierce) were added to cell lysates and the mixture was rotated at $4^{\circ} \mathrm{C}$ for 2 hours. Avidin beads were washed twice with ice-cold lysis buffer and biotinylated proteins were extracted with SDS sample buffer containing $50 \mathrm{mM}$ of fresh DTT for $5 \mathrm{~min}$ at $95^{\circ} \mathrm{C}$. Biotinylated GluA1 and total GluA1 (cell lysate) were then probed with $\alpha$ GluA1 and detected with HRP-labeled secondary Abs using Chemidoc (Bio-Rad).

\section{Results}

\section{Impaired LTP and LTD in Stim2 cKO mouse}

We crossed mice homozygous for the LoxP-flanked (floxed, fl) Stim2 allele (Stim2 ${ }^{f / f l}$ ) ${ }^{25}$ with a CaMKII $\alpha$-Cre transgenic line ${ }^{26}$ to conditionally inactive the Stim 2 gene in excitatory neurons of the forebrain. Analysis of hippocampal lysates prepared from Stim2 ${ }^{\mathrm{ffl} / \mathrm{ll}} ; \mathrm{CaMKII} \alpha-\mathrm{Cre}^{+}$(Stim2 cKO) adult mice showed a marked decrease in STIM2 expression compared to their control 
$\left(\right.$ Stim2 $2^{\mathrm{fl} / \mathrm{fl}} ;$ CaMKII $\alpha$-Cre ${ }^{-}$) littermates (Figure 1), reflecting efficient Cre-mediated excision of the floxed Stim2 gene. Expression of STIM1 or GluA1 was unaffected by ablation of the Stim2 gene (Figure 1). Further examination of these mice revealed no apparent defects in brain anatomy and neuropil density in the cortex and hippocampus (not shown, but see ${ }^{12}$ ).

We next measured LTP at CA3-CA1 hippocampal synapses in adult ( 3 months old) Stim2 cKO and control littermates. We chose to induce a protein synthesis-independent form of LTP (Early-LTP, E-LTP) which is largely driven by synaptic incorporation of GluA1-containing AMPARs ${ }^{17,29}$. E-LTP was induced by a weak tetanization protocol (WTET) consisting of a single high-frequency stimulation of Schaffer collaterals ${ }^{30,31}$. This stimulation protocol led to robust potentiation of synaptic strength in control mice, which persisted up to 2 hours after induction (Figure 2A). In contrast, E-LTP in Stim2 cKO mice rapidly declined and fEPSPs fell to baseline levels about 90 min after induction (Figure 2A). Measurement of E-LTD, in response to a weak low frequency stimulation protocol (WLFS) ${ }^{31,32}$ produced similar results. LTD was not stably maintained in Stim2 cKO mice beyond $\sim 40 \mathrm{~min}$, while it persisted up to $90 \mathrm{~min}$ in control littermates (Figure 2B). Thus, STIM2 exerts profound effects on synaptic plasticity in the hippocampus.

\section{STIM2 regulates dendritic spine shape and density}

Because synaptic plasticity is linked to the micro-architecture of dendritic spines, we analyzed the size, shape and density of dendritic spines in apical dendrites of CA1 pyramidal cells, which receive inputs from Schaffer collateral axons. For this, we stereotactically introduced a GFPexpressing lentivirus in the CA1 region of Stim $2 \mathrm{cKO}$ mice or control littermates (Figure 3A,B) and analyzed several thousand spines for each genotype, in $\sim 3$ months old animals. We observed a mild but significant decrease in both the density and size of dendritic spines in Stim2 cKO mice (Figure C-E). Moreover, Stim2-depleted CA1 neurons show fewer "mushroom" spines and a corresponding increase in "stubby" spines, while the fraction of "thin" spines is unaltered (Figure 3F). These results are similar, though not identical (see discussion), to a previous study describing spine defects in CA1 pyramidal neurons from Stim $2^{f l / f l}$ mice virally transduced with $\mathrm{Cre}^{8}$. As excision of the Stim2 gene occurs in adult Stim2 cKO mice after spinogenesis, this deficit in mushroom spines most likely reflects a failure in the maintenance of mature spines rather than a developmental defect. It has been proposed that stable mushroom 
spines store information over long-period of times ${ }^{33}$. Reduced number of mushroom spines in Stim2-depleted pyramidal cells is therefore consistent with impaired plasticity and information processing in these mice. Whether this spine structural defect is a cause or consequence of impaired hippocampal plasticity in Stim2 cKO animals is not known at this time.

\section{Pleiotropic effects of STIM2 on GluA1 trafficking.}

In the adult hippocampus, the AMPAR consists mainly of GluA1/GluA2 and, to a lesser extent, GluA2/GluA3 hetero-tetramers ${ }^{34}$. GluA1 mediates activity-dependent synaptic targeting and removal of the AMPAR during LTP ${ }^{17,19}$ and LTD ${ }^{14}$, in part through phosphorylation and dephosphorylation of its cytoplasmic domain ${ }^{35}$. We therefore examined trafficking of GluA1 in neurons from Stim $2^{f l f l}$ mice transduced with mCherry-P2A-Cre, or mCherry only (as a control) using a surface biotinylation technique ${ }^{7,36}$. We chose to perform these experiments in cortical neuron cultures, as these preparations yield enough cells for biochemistry. Titers of mCherryP2A-Cre lentiviruses were adjusted in order to obtain efficient ablation of the Stim2 gene in these neuron cultures (Figure 4A). We first measured GluA1 surface expression in response to a chemical LTP (cLTP) protocol based on cAMP elevation by forskolin/rolipram (forsk-LTP). Forsk-LTP triggers GluA1 exocytosis through PKA phosphorylation of its C-terminal tail on Ser845 ${ }^{13,36}$. In control (Cre -) Stim $2^{f l / f l}$ neurons, forsk-LTP induced robust surface delivery of GluA1 (Figure 4B). In Stim2 cKO neurons, however, basal surface expression of GluA1 was slightly elevated and forsk-LTP failed to increase surface expression of GluA1. In fact, GluA1 surface levels after forsk-LTP were lower than in basal conditions and comparable to those observed in resting control cells. A similar result was observed in response to a different cLTP protocol (glyc-LTP) that relies on spontaneous glutamate release (see methods). Reduced GluA1 surface expression was observed after glyc-LTP in Stim 2 cKO neurons, while the same treatment led to a marked augmentation of surface GluA1 in control cells (Figure 4C). Thus, Stim2 cKO neurons respond to two distinct cLTP protocols by reducing surface expression of GluA1, a behavior which is fundamentally opposite to the normal situation and likely to affect both LTP and LTD (see discussion).

We then evaluated the role of Stim2 in GluA1 endocytosis by bath application of NMDA, a treatment known to induce rapid AMPAR endocytosis and synaptic depression ${ }^{37}$. Whereas NMDA triggered robust removal of GluA1 from the cell surface in control cells, it had, 
remarkably, no effect on GluA1 surface levels in Stim2 cKO neurons (Figure 4D), indicating an essential function of STIM2 in activity-dependent GluA1 endocytosis. Thus, STIM2 is required for exo- and endocytosis of GluA1 in response to pharmacological manipulations that mimic LTP and LTD. The pronounced influence of STIM2 on activity-dependent GluA1 trafficking likely underlies the plasticity deficits observed in Stim2 cKO mice.

\section{Discussion}

We report here a central role of STIM2 in the expression of early forms of LTP and LTD at hippocampal CA1-Schaffer collateral synapses. The impact of STIM2 on both spine morphogenesis and AMPAR trafficking provides insights into the mechanisms by which this ER signaling protein regulates activity-dependent changes in synaptic efficacy.

The expression of E-LTP at CA3-CA1 hippocampal synapses critically depends on activitydependent incorporation of GluA1-containing AMPARs in the post-synaptic membrane ${ }^{35}$. Activity-dependent synaptic targeting of AMPARs is driving by lateral movement of extrasynaptic AMPARs to the synapse and by exocytosis of an intracellular pool of AMPARs ${ }^{38}$. The pronounced deficit in GluA1 surface delivery we observed in Stim2 cKO neurons, in response to two different cLTP protocols, points to a central role of STIM2 in regulating AMPAR trafficking, and suggests that abnormal AMPAR trafficking during plasticity underlies E-LTP defects in Stim2 cKO mice. PKA phosphorylation of GluA1 on Ser845 has now been shown by many groups to stimulate surface delivery and synapse incorporation of AMPARs ${ }^{13,14,36,37,39,40}$. We previously reported that PKA phosphorylation of GluA1 depends on STIM2 ${ }^{7,12}$ and is driving by the assembly of a PKA signaling complex at contact sites between the ER and the dendritic plasma membrane ${ }^{7}$. Regulation of GluA1 Ser845 phosphorylation is therefore one plausible mechanism by which STIM2 promotes synaptic targeting of AMPARs during LTP.

LTD is mediated by endocytic removal of AMPARs from the synapse. Inhibition of NMDAinduced GluA1 endocytosis in Stim2 cKO neurons is, therefore, consistent with reduced E-LTD in these animals. How STIM2 regulates GluA1 endocytosis in response to LTD-inducing stimuli is at present unknown, but it is conceivable that the underlying mechanism also involves PKA signaling and regulation of GluA1 Ser845 phosphorylation. Arguably the strongest evidence for 
a role of GluA1 p-Ser845 in LTD came from a knock-in mouse with a Serine to Alanine mutation at this site. This Ser845 phospho-mutant mouse exhibits LTD deficits at CA3-CA1 synapses that are strikingly similar to those observed in Stim2 cKO animals ${ }^{41}$. Similar LTD deficits have been reported in AKAP79/150 KO mice, a PKA-anchoring protein that regulates GluA1 phosphorylation on Ser845 ${ }^{42}$. Moreover, endocytosis of GluA1 in response to bath application of NMDA is associated with dephosphorylation of GluA1 on Ser845 ${ }^{36,37}$. Taken together, these results suggest that both phosphorylation and dephosphorylation of GluA1 on Ser845 is important for the expression of LTD at hippocampal synapses. Based on these data, and our earlier work describing a critical role of STIM2 in PKA phosphorylation of GluA1 7,12, we propose that the impact of STIM2 on GluA1 endocytosis and E-LTD is mediated, at least in part, through regulation of GluA1 Ser845 phosphorylation. The influence of STIM2 on AMPAR dynamics is most certainly not restricted to its effects on GluA1 phosphorylation. Whether SOCE, or other effector pathways contribute to STIM2-dependent regulation of AMPAR trafficking and plasticity remains to be established.

The spine deficits we observed in adult Stim2 cKO mice - decrease in spine density and reduced fraction of mushroom spines in CA1 pyramidal cells - are analogous, though slightly different than these previously reported by another group. Using the same Stim2 $2^{f / f l}$ mouse, stereotactically injected with AAV-Cre in CA1, Sun et al., reported a decrease in mushroom spines with no effect on spine density ${ }^{8}$. The reason for this minor discrepancy is not clear. Both studies, however, confirm a role of STIM2 in the maintenance of mature, mushroom-like spines in the adult brain. Of interest, STIM2 preferentially localizes to large spines ${ }^{7}$ and its distribution mirrors that of the ER itself, which is present in a subset of spines ( $\sim 20 \%$ to $50 \%$ depending on the cell type) with large heads ${ }^{43}$. ER-containing spines exhibit strong synaptic currents ${ }^{43}$ and display a form of LTD which is mGluR-dependent ${ }^{43}$. Although we have not directly investigated mGluR-LTD in this study, these results suggest that STIM2 preferentially regulates bidirectional plasticity of large spines containing strong synapses. Direct evidence for a role of STIM2 in structural plasticity will require live-imaging of spine dynamics in Stim2 cKO mice in response to LTP- or LTD-inducing protocols. 
In conclusion, we identified STIM2 as a novel regulator of early forms of LTP and LTD in the hippocampus. STIM2 signaling might also take part, however, in persistent forms of plasticity, such as protein synthesis-dependent late LTP or LTD, a possibility that we will investigate in future studies. Our results provide new insights on how the ER interacts with and modifies the properties of excitatory synapses and hint at a key role of ER-to-synapse signaling in activitydependent changes of synaptic efficacy. 


\section{References}

1 Mattson, M. P. et al. Calcium signaling in the ER: its role in neuronal plasticity and neurodegenerative disorders. Trends Neurosci 23, 222-229, doi:S0166-2236(00)01548-4 [pii] (2000).

2 Bardo, S., Cavazzini, M. G. \& Emptage, N. The role of the endoplasmic reticulum Ca2+ store in the plasticity of central neurons. Trends Pharmacol Sci 27, 78-84, doi:10.1016/j.tips.2005.12.008 (2006).

3 Carrasco, S. \& Meyer, T. STIM proteins and the endoplasmic reticulum-plasma membrane junctions. Annu Rev Biochem 80, 973-1000, doi:10.1146/annurev-biochem061609-165311 (2011).

4 Parekh, A. B. \& Putney, J. W., Jr. Store-operated calcium channels. Physiol Rev 85, 757810, doi:10.1152/physrev.00057.2003 (2005).

5 Park, C. Y., Shcheglovitov, A. \& Dolmetsch, R. The CRAC channel activator STIM1 binds and inhibits L-type voltage-gated calcium channels. Science 330, 101-105, doi:10.1126/science.1191027

330/6000/101 [pii] (2010).

6 Arakawa, N. et al. KB-R7943 inhibits store-operated $\mathrm{Ca}(2+)$ entry in cultured neurons and astrocytes. Biochem Biophys Res Commun 279, 354-357, doi:10.1006/bbrc.2000.3968 (2000).

7 Garcia-Alvarez, G. et al. STIM2 regulates PKA-dependent phosphorylation and trafficking of AMPARs. Mol Biol Cell, doi:10.1091/mbc.E14-07-1222 (2015).

8 Sun, S. et al. Reduced Synaptic STIM2 Expression and Impaired Store-Operated Calcium Entry Cause Destabilization of Mature Spines in Mutant Presenilin Mice. Neuron 82, 7993, doi:10.1016/j.neuron.2014.02.019 (2014).

9 Popugaeva, E. et al. STIM2 protects hippocampal mushroom spines from amyloid synaptotoxicity. Mol Neurodegener 10, 37, doi:10.1186/s13024-015-0034-7 (2015).

$10 \mathrm{Wu}$, J. et al. Enhanced Store-Operated Calcium Entry Leads to Striatal Synaptic Loss in a Huntington's Disease Mouse Model. J Neurosci 36, 125-141, doi:10.1523/JNEUROSCI.1038-15.2016 (2016). 
11 Zhang, H. et al. Neuronal Store-Operated Calcium Entry and Mushroom Spine Loss in Amyloid Precursor Protein Knock-In Mouse Model of Alzheimer's Disease. J Neurosci 35, 13275-13286, doi:10.1523/JNEUROSCI.1034-15.2015 (2015).

12 Garcia-Alvarez, G. et al. Impaired spatial memory and enhanced long-term potentiation in mice with forebrain-specific ablation of the Stim genes. Frontiers in behavioral neuroscience 9, 180, doi:10.3389/fnbeh.2015.00180 (2015).

13 Oh, M. C., Derkach, V. A., Guire, E. S. \& Soderling, T. R. Extrasynaptic membrane trafficking regulated by GluR1 serine 845 phosphorylation primes AMPA receptors for long-term potentiation. J Biol Chem 281, 752-758, doi:M509677200 [pii]

10.1074/jbc.M509677200 (2006).

14 Esteban, J. A. et al. PKA phosphorylation of AMPA receptor subunits controls synaptic trafficking underlying plasticity. Nat Neurosci 6, 136-143, doi:10.1038/nn997

nn997 [pii] (2003).

15 Banke, T. G. et al. Control of GluR1 AMPA receptor function by cAMP-dependent protein kinase. J Neurosci 20, 89-102 (2000).

16 Wang, Y. et al. The calcium store sensor, STIM1, reciprocally controls Orai and CaV1.2 channels. Science 330, 105-109, doi:10.1126/science.1191086

330/6000/105 [pii] (2010).

17 Zamanillo, D. et al. Importance of AMPA receptors for hippocampal synaptic plasticity but not for spatial learning. Science 284, 1805-1811 (1999).

18 Hayashi, Y. et al. Driving AMPA receptors into synapses by LTP and CaMKII: requirement for GluR1 and PDZ domain interaction. Science 287, 2262-2267 (2000).

19 Shi, S., Hayashi, Y., Esteban, J. A. \& Malinow, R. Subunit-specific rules governing AMPA receptor trafficking to synapses in hippocampal pyramidal neurons. Cell 105, 331-343 (2001).

20 Matsuzaki, M., Honkura, N., Ellis-Davies, G. C. \& Kasai, H. Structural basis of longterm potentiation in single dendritic spines. Nature 429, 761-766, doi:10.1038/nature02617

nature02617 [pii] (2004). 
21 Zhou, Q., Homma, K. J. \& Poo, M. M. Shrinkage of dendritic spines associated with long-term depression of hippocampal synapses. Neuron 44, 749-757, doi:10.1016/j.neuron.2004.11.011 (2004).

22 Skibinska-Kijek, A., Wisniewska, M. B., Gruszczynska-Biegala, J., Methner, A. \& Kuznicki, J. Immunolocalization of STIM1 in the mouse brain. Acta Neurobiol Exp (Wars) 69, 413-428, doi:6930 [pii] (2009).

23 Tashiro, A., Zhao, C. \& Gage, F. H. Retrovirus-mediated single-cell gene knockout technique in adult newborn neurons in vivo. Nat Protoc 1, 3049-3055, doi:10.1038/nprot.2006.473 (2006).

24 Tiscornia, G., Singer, O. \& Verma, I. M. Production and purification of lentiviral vectors. Nat Protoc 1, 241-245, doi:nprot.2006.37 [pii]

10.1038/nprot.2006.37 (2006).

25 Oh-Hora, M. et al. Dual functions for the endoplasmic reticulum calcium sensors STIM1 and STIM2 in T cell activation and tolerance. Nat Immunol 9, 432-443, doi:ni1574 [pii]

10.1038/ni1574 (2008).

26 Tsien, J. Z. et al. Subregion- and cell type-restricted gene knockout in mouse brain. Cell 87, 1317-1326 (1996).

27 Madisen, L. et al. A robust and high-throughput Cre reporting and characterization system for the whole mouse brain. Nat Neurosci 13, 133-140, doi:10.1038/nn.2467 (2010).

28 Rodriguez, A., Ehlenberger, D. B., Dickstein, D. L., Hof, P. R. \& Wearne, S. L. Automated three-dimensional detection and shape classification of dendritic spines from fluorescence microscopy images. PLoS One 3, e1997, doi:10.1371/journal.pone.0001997 (2008).

29 Derkach, V. A., Oh, M. C., Guire, E. S. \& Soderling, T. R. Regulatory mechanisms of AMPA receptors in synaptic plasticity. Nat Rev Neurosci 8, 101-113, doi:10.1038/nrn2055 (2007).

30 Frey, U. \& Morris, R. G. Weak before strong: dissociating synaptic tagging and plasticity-factor accounts of late-LTP. Neuropharmacology 37, 545-552 (1998).

31 Sajikumar, S., Navakkode, S. \& Frey, J. U. Identification of compartment- and processspecific molecules required for "synaptic tagging" during long-term potentiation and 
long-term depression in hippocampal CA1. J Neurosci 27, 5068-5080, doi:10.1523/JNEUROSCI.4940-06.2007 (2007).

32 Sajikumar, S. \& Frey, J. U. Anisomycin inhibits the late maintenance of long-term depression in rat hippocampal slices in vitro. Neurosci Lett 338, 147-150 (2003).

33 Bourne, J. \& Harris, K. M. Do thin spines learn to be mushroom spines that remember? Curr Opin Neurobiol 17, 381-386, doi:10.1016/j.conb.2007.04.009 (2007).

$34 \mathrm{Lu}, \mathrm{W}$. et al. Subunit composition of synaptic AMPA receptors revealed by a single-cell genetic approach. Neuron 62, 254-268, doi:10.1016/j.neuron.2009.02.027 (2009).

35 Huganir, R. L. \& Nicoll, R. A. AMPARs and synaptic plasticity: the last 25 years. Neuron 80, 704-717, doi:10.1016/j.neuron.2013.10.025 (2013).

36 Man, H. Y., Sekine-Aizawa, Y. \& Huganir, R. L. Regulation of \{alpha\}-amino-3hydroxy-5-methyl-4-isoxazolepropionic acid receptor trafficking through PKA phosphorylation of the Glu receptor 1 subunit. Proc Natl Acad Sci U S A 104, 3579-3584, doi:0611698104 [pii]

10.1073/pnas.0611698104 (2007).

37 Ehlers, M. D. Reinsertion or degradation of AMPA receptors determined by activitydependent endocytic sorting. Neuron 28, 511-525, doi:S0896-6273(00)00129-X [pii] (2000).

38 Makino, H. \& Malinow, R. AMPA receptor incorporation into synapses during LTP: the role of lateral movement and exocytosis. Neuron 64, 381-390, doi:10.1016/j.neuron.2009.08.035

S0896-6273(09)00675-8 [pii] (2009).

39 Lee, H. K. et al. Phosphorylation of the AMPA receptor GluR1 subunit is required for synaptic plasticity and retention of spatial memory. Cell 112, 631-643, doi:S0092867403001223 [pii] (2003).

40 Makino, Y., Johnson, R. C., Yu, Y., Takamiya, K. \& Huganir, R. L. Enhanced synaptic plasticity in mice with phosphomimetic mutation of the GluA1 AMPA receptor. Proc Natl Acad Sci U S A 108, 8450-8455, doi:10.1073/pnas.1105261108 1105261108 [pii] (2011).

41 Lee, H. K., Takamiya, K., He, K., Song, L. \& Huganir, R. L. Specific roles of AMPA receptor subunit GluR1 (GluA1) phosphorylation sites in regulating synaptic plasticity in 
the CA1 region of hippocampus. J Neurophysiol 103, 479-489, doi:10.1152/jn.00835.2009

00835.2009 [pii] (2010).

42 Tavalin, S. J. et al. Regulation of GluR1 by the A-kinase anchoring protein 79 (AKAP79) signaling complex shares properties with long-term depression. J Neurosci 22, 30443051, doi:20026277 (2002).

43 Holbro, N., Grunditz, A. \& Oertner, T. G. Differential distribution of endoplasmic reticulum controls metabotropic signaling and plasticity at hippocampal synapses. Proc Natl Acad Sci U S A 106, 15055-15060, doi:10.1073/pnas.0905110106 0905110106 [pii] (2009). 
Acknowledgements. We thank Tan Li Ting and Durgadevi Alagappan for excellent technical assistance. This work was supported by grants to MF from the Singapore Ministry of Education Academic Research Fund (MOE2011-T2-1-107), and the Agency for Science, Technology and Research (Astar/JJSI 13/1/24/26/011). SS is supported by the National Medical Research Council, Singapore (NMRC-CBRG-0041/2013).

Author contribution. KAFY performed the stereotactic lentiviral delivery and imaging experiments. MSS did the LTP and LTD experiments. GGA performed all the biochemistry. SS designed and supervised the electrophysiological component of the project. MO generated the floxed Stim2 mice. MF designed and supervesised the project and BL and MF wrote the manuscript.

Disclosure of competing interests. The authors declare no conflict of interest. 


\section{Figure legends}

Figure 1. Ablation of STIM2 in the hippocampus of Stim2 cKO mice. Immunoblots of hippocampal lysates prepared from 3 month-old Stim2 $2^{\mathrm{fl} / \mathrm{fl}} ;$ CaMKII $\alpha-C r e^{-}$and Stim2 ${ }^{\mathrm{fl} / \mathrm{fl}} ;$ CaMKII $\alpha$ $\mathrm{Cre}^{+}$littermates and probed with the indicated antibodies. All immunoblots were performed on the same Stim2 $2^{\mathrm{fl} / \mathrm{fl} l} ;$ CaMKII $\alpha-C r e^{-}$and Stim2 $2^{\mathrm{fl} / \mathrm{fl}} ;$ CaMKII $\alpha$-Cre ${ }^{+}$samples. Shown here are results from one animal of each genotype. See ${ }^{12}$ for a quantitative analysis of STIM2 levels in Stim2 $\mathrm{cKO}$ mice, in different brain regions.

Figure 2. Impaired synaptic plasticity in Stim2 cKO mice. (A) Weak tetanisation (WTET)induced LTP recorded in CA1 from Stim $2^{f l / f l} ; \mathrm{Cre}^{+}(n=7$ slices from 5 mice) mice and their control $\left(\mathrm{Cr}^{-}\right)$littermates ( $n=7$ slices from 4 mice). Potentiation in $\mathrm{Cr}^{-}$group is significantly different from the baseline values until $155 \min (+155 \mathrm{~min}, p<0.05$; Wilcoxon matched pairs sign test) whereas in $\mathrm{Cr} e^{+}$group it is significantly different only up to $45 \min (+45 \mathrm{~min}, p<0.05$; Wilcoxon matched pairs sign test). When compared between the groups, the potentiation is significantly different between the two groups up to 115 minutes beyond which it was not significant ( $p>0.05$; Mann-Whitney U test). (B) Weak low frequency stimulation (WLFS)induced E-LTD recorded in CA1 from Stim2 $2^{f l f l} ; \mathrm{Cre}^{+}(n=6$ slices from 3 mice) mice and their control $\left(\mathrm{Cr}^{+}\right)$littermates ( $n=6$ slices from 3 mice). Depression in $\mathrm{Cr}^{-}$group is significantly different from the pre-induction values until 80 minutes ( $+80 \mathrm{~min}, p<0.05$; Wilcoxon matched pairs sign test) whereas in $C r e^{+}$group the depression was significant only up to 40 minutes $(+40$ min, $p<0.05$; Wilcoxon matched pairs sign test). The mean depression immediately after WLFS in $\mathrm{Cr}^{+}$group $(72.75 \pm 4.06 \%)$ is significantly different than that in $C r e^{-}$group $(60.33 \pm 4.44 \%)$; Mann-Whitney $\mathrm{U}$ test, $p<0.05$. The insets show representative fEPSP traces recorded at the specified times before and after stimulation. Data are plotted as mean $\pm \mathrm{SEM}$.

Figure 3. Reduction in spine density and head diameter in Stim2 cKO mice. (A) Coronal section of adult mouse ( 3 months old) stereotactically injected with pFUGW lentiviruses and immunostained for GFP. Right panel is a magnification of the area indicated in the left panel and shows a single pyramidal neuron in the CA1 region of the hippocampus. (B) Confocal imaging of dendritic spines in apical dendrites of CA1 hippocampal neurons from Stim2 ${ }^{\text {flfl }}$;Cre ${ }^{+}$(cKO) and $\mathrm{Cr}^{-}$(control). Scale bars represent $5 \mu \mathrm{m}$. (C) Average spine density, (D) cumulative 
frequency of spine density and (E) average spine head diameter in CA1 neurons of Stim $2^{f / f f}$; $\mathrm{Cre}^{+}$ (4259 spines, 83 dendritic segments) and $\mathrm{Cre}^{-}$(5988 spines, 105 dendritic segments) mice. ***p $<0.001$ by t-test. (F) Percentage of mushroom, stubby, and thin spines types in CA1 hippocampal neurons from Stim $2^{f / / f l} ; \mathrm{Cre}^{+}$and $\mathrm{Cre}^{-}$mice. ${ }^{* * *} \mathrm{p}<0.001$ by one-way ANOVA followed by post-hoc Bonferroni test. $\mathrm{N}=3$ mice for each group. Values are shown as Mean \pm SEM.

Figure 4. STIM2 regulates exo- and endocytosis of GluA1. (A) Stim2 immunoblot from Stim $2^{f / f l}$ cortical neurons (DIV21) transduced with mCherry-P2A-Cre (Cre + ) or mCherry (Cre -). (B-D). Stim2 $2^{f / f l}$ cortical neurons (DIV21) transduced with mCherry-P2A-Cre (Cre +) or mCherry (Cre -) and exposed to (B) forks-LTP (30 min), (C) glyc-LTP (15 min) and (D) NMDA-LTD ( $3 \mathrm{~min})$ at $37^{\circ} \mathrm{C}$. Surface (biotinylated) and total GluA1 were analyzed by immunoblotting. Average surface GluA1 was quantified by densitometry from 3 independent experiments. Error bars indicate standard deviation from the mean. Blots were vertically cropped in (B) to remove an empty lane. 
Yap_Fig.1

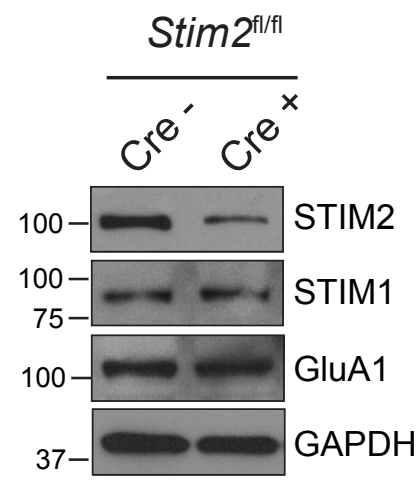


A

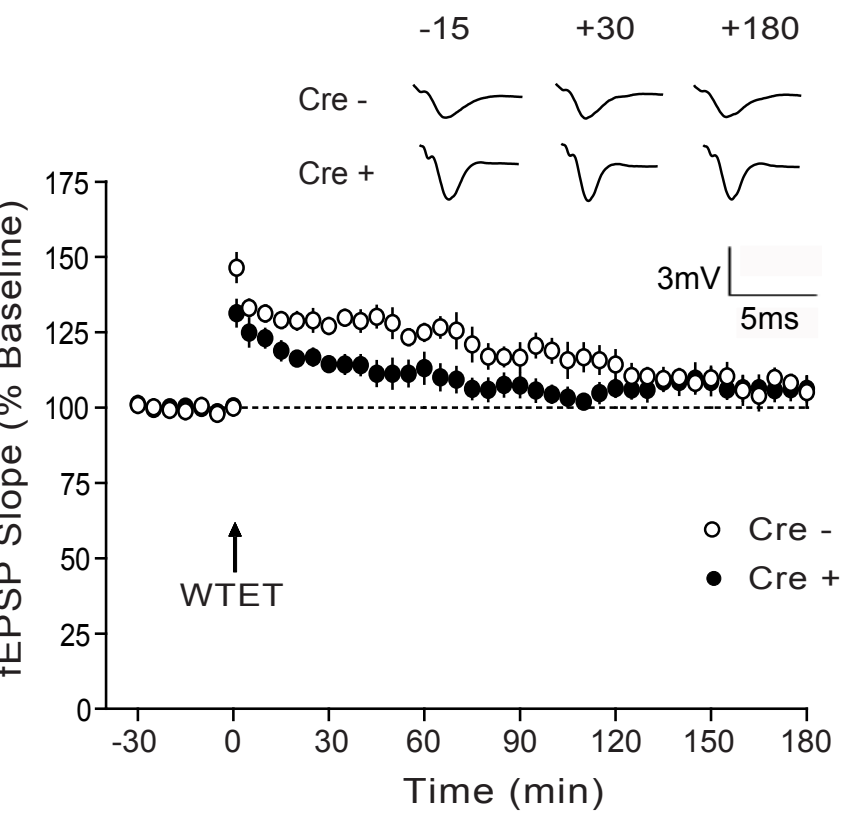

B
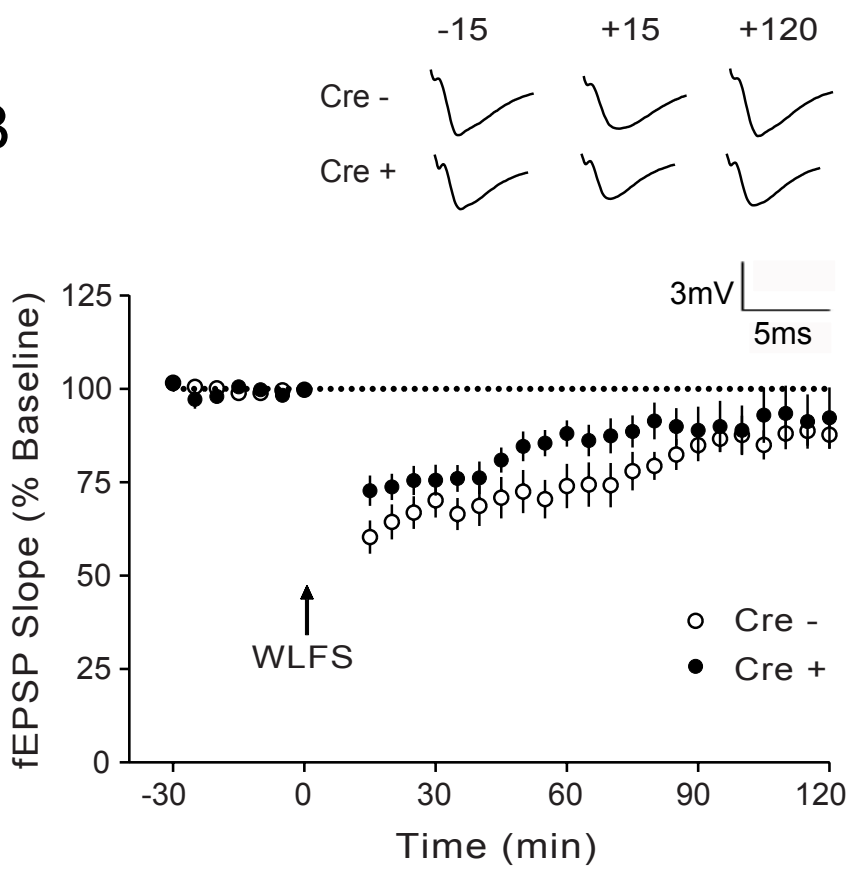
A

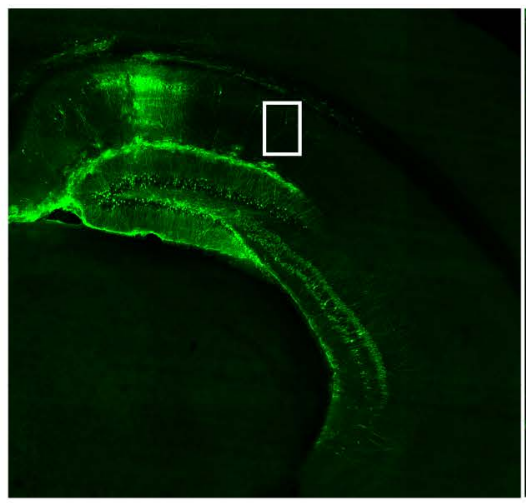

C

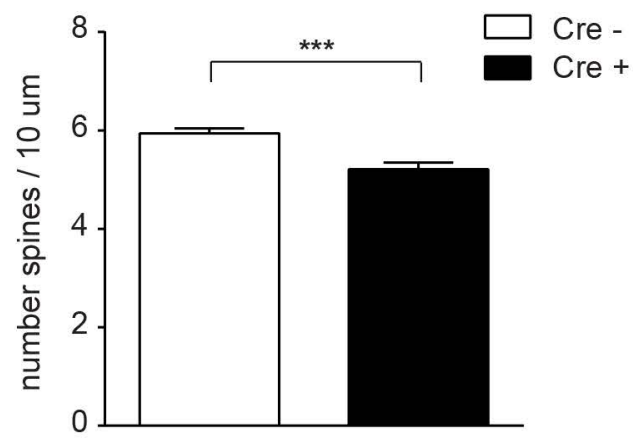

E

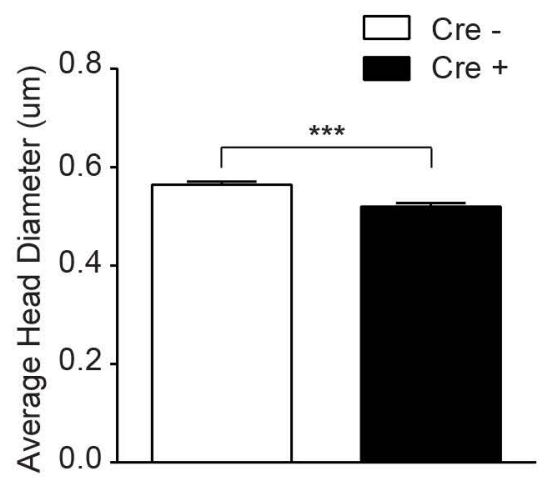

B

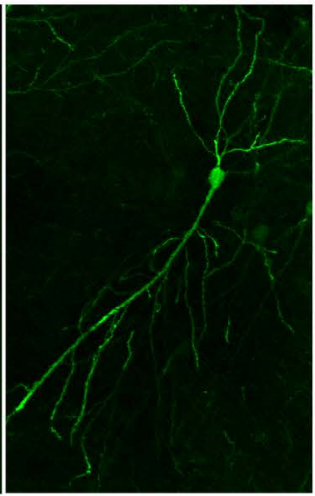

Cre -
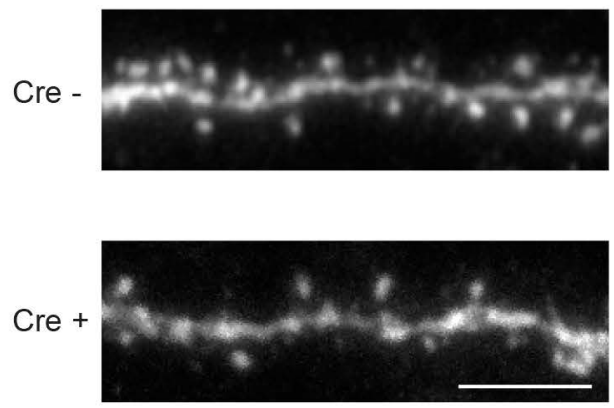

D

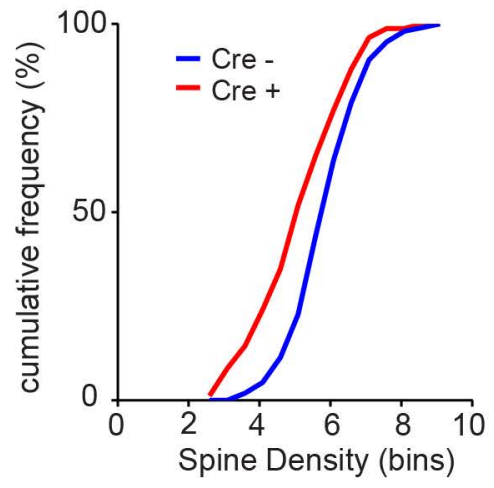

F

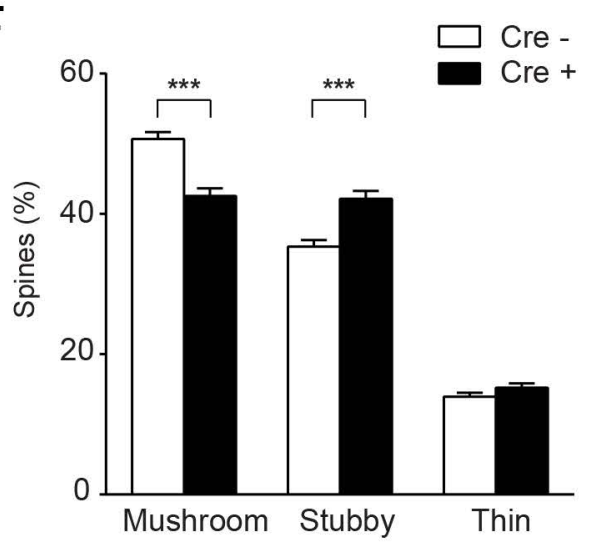



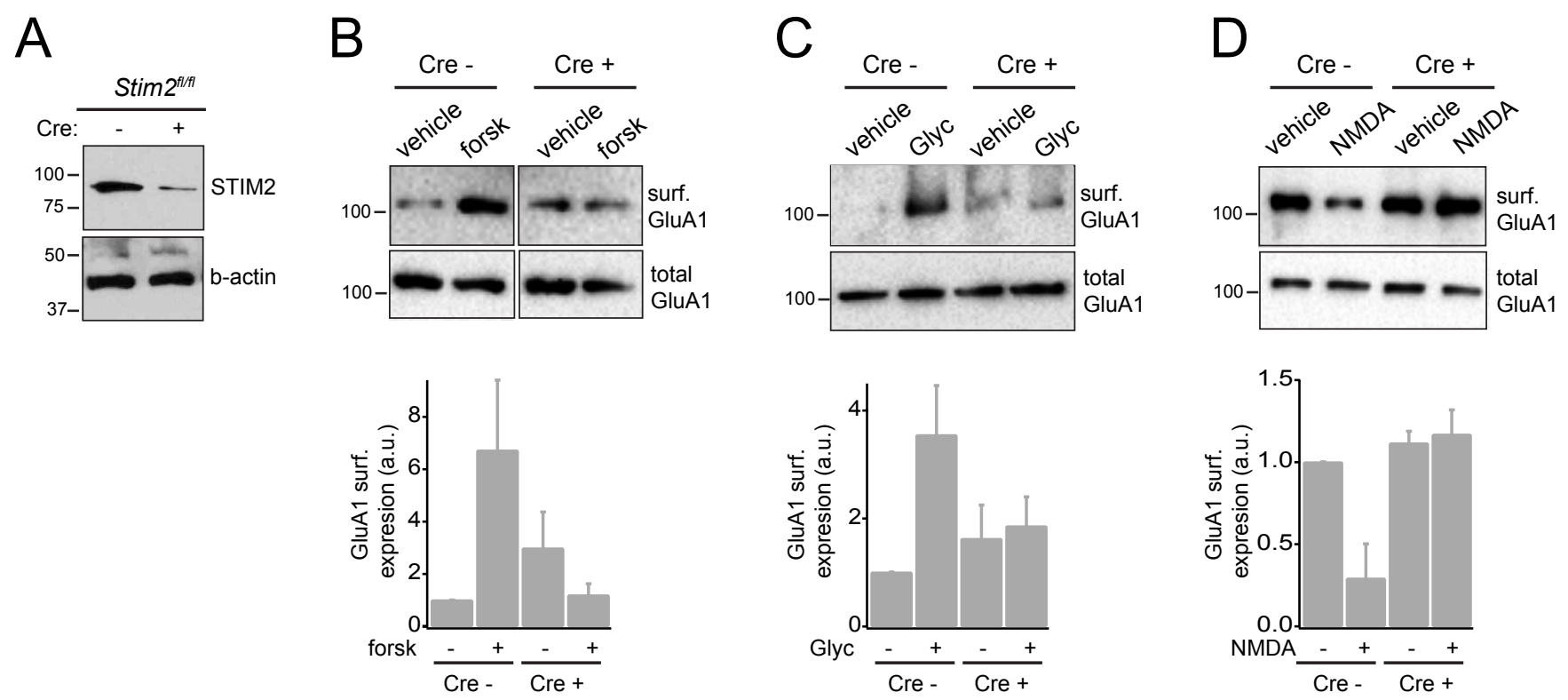